\title{
Timing and site-specific trends of recurrence in patients with pathological stage II or III gastric cancer after curative gastrectomy followed by adjuvant S-1 monotherapy
}

\author{
Ryo Takahashi ${ }^{1,2} \cdot$ Manabu Ohashi $^{1}$ (D) Yosuke Kano $^{1} \cdot$ Satoshi Ida ${ }^{1} \cdot$ Koshi Kumagai $^{1} \cdot$ Souya Nunobe ${ }^{1} \cdot$ Keisho Chin $^{1}$. \\ Kensei Yamaguchi ${ }^{1} \cdot$ Masato Nagino ${ }^{2} \cdot$ Takeshi Sano $^{1} \cdot$ Naoki Hiki $^{1}$
}

Received: 27 December 2018 / Accepted: 11 March 2019 / Published online: 15 March 2019

(c) The International Gastric Cancer Association and The Japanese Gastric Cancer Association 2019

\begin{abstract}
Background Adjuvant S-1 monotherapy prolongs the survival of patients with pathological stage II or III gastric cancer undergoing D2 gastrectomy. This therapeutic regimen is standard in Japan. Unfortunately, some patients who undergo this treatment suffer from recurrent disease. However, information regarding the timing and site-specific trends of recurrence is insufficient.

Methods Among 396 patients who underwent D2 gastrectomy followed by adjuvant S-1 monotherapy between 2008 and 2012, 122 experienced a recurrence. We retrospectively determined the timing and sites of recurrence.

Results The median RFS of the 122 patients was 19.5 months, and their 1-, 3- and 5-year RFS rates were 67.2\%, 23.0\% and $5.7 \%$, respectively. There were no significant differences in RFS among disease substages. Local recurrence, lymph node involvement and peritoneal and hematogenous metastases were found in 6, 25, 63 and 42 patients, respectively. Approximately $10 \%$ of patients presented with contemporaneous sites of recurrence. Local recurrence and lymph node metastasis plateaued 3 years after gastrectomy. Peritoneal and hematogenous metastasis increased within 5 years after surgery. In patients with hematogenous metastasis, the number of liver metastases plateaued but increased in others.

Conclusions In patients with recurrent disease who underwent D2 gastrectomy followed by adjuvant S-1 monotherapy, $80 \%$ of recurrences occur within 3 years after gastrectomy. The timing of recurrence is not significantly different among substages. Although the rates of local recurrence and lymph node and liver metastasis plateau after 3 years, peritoneal and the other hematogenous metastases increase within 5 years.
\end{abstract}

Keyword Gastric cancer $\cdot$ S-1 $\cdot$ Adjuvant chemotherapy $\cdot$ Recurrence $\cdot$ Timing $\cdot$ Site-specific trends

Ryo Takahashi and Manabu Ohashi contributed equally to this study.

Manabu Ohashi

manabu.ohashi@jfcr.or.jp

1 Department of Gastroenterology, Cancer Institute Hospital, Japanese Foundation for Cancer Research, 3-8-31, Ariake, Koto-ku, Tokyo 135-8550, Japan

2 Division of Surgical Oncology, Department of Surgery, Nagoya University Graduate School of Medicine, Nagoya, Japan

\section{Introduction}

Gastric cancer is the third leading cause of cancer-related death and the fifth most common cancer diagnosed worldwide, affecting approximately 1 million new individuals each year and causing at least 700,000 deaths [1]. Although early gastric cancer is typically curable, reported that 5-year overall survival (OS) rates was $91.7 \%$ [2], locally advanced disease sometimes relapses, even in patients who undergo radical gastrectomy. The Adjuvant Chemotherapy Trial of S-1 for Gastric Cancer (ACTS-GC) was the first to reveal the benefit of adjuvant chemotherapy $[3,4]$. Specifically, this phase III, multi-center randomized control trial demonstrated that S-1 significantly improves the rates of OS and relapse-free survival (RFS) of patients with pathological stage (pStage) II or III gastric cancer. 
The results of this nationwide clinical trial, conducted in Japan, led to the adoption in 2007 of adjuvant S-1 monotherapy as the standard treatment for pStage II or III gastric cancer [5].

To prolong the survival periods of patients with recurrent gastric cancer, postoperative surveillance is an important component of treatments for gastric cancer, along with surgery and adjuvant chemotherapy. Knowledge of the OS or RFS of each stage is critically important for the detection of recurrence during follow-up and for treatment intervention after recurrence [6]. Moreover, data regarding timing and site-specific trends of recurrence are sometimes more helpful as guides for conducting effective follow-up examinations. For example, before the implementation of adjuvant S-1 monotherapy, approximately $80 \%$ of recurrences were detected within 2 years after radical gastrectomy for locally advanced gastric cancer [7,8]. Although effective adjuvant S-1 monotherapy may further prolong the interval between surgery and recurrence [9], to our knowledge, published supporting data are lacking.
Here, we conducted a retrospective analysis of the timing of recurrence and site-specific trends experienced by patients with pStage II or III gastric cancer who received adjuvant S-1 monotherapy following gastrectomy with D2 lymph node dissection. This is the first report, to our knowledge, focusing on the surveillance strategy. This information will provide a foundation for implementing systematic and effective surveillance strategies.

\section{Methods}

\section{Patients}

We reviewed the records of 550 patients with pStage II or III gastric cancer, excluding pT1 and pT3N0, who underwent curative D2-distal or total gastrectomy at the Cancer Institute Hospital, Tokyo, Japan between 2008 and 2012 (Fig. 1). We excluded 47 patients who underwent less than D2 lymph node dissection, with remnant gastric cancer or those who
Fig. 1 Patient selection
Patients who were diagnosed with pathologic stage II-III (excluded T3N0, T1N2-3) gastric cancer after radical standard gastrectomy $(n=550)$

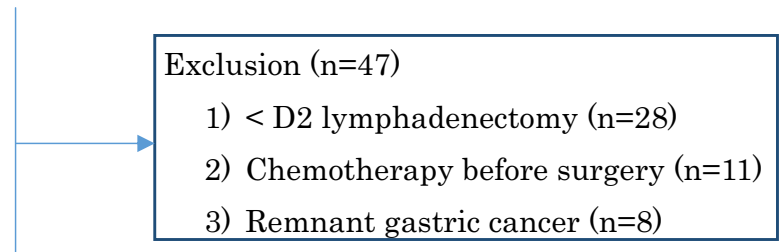

Patients eligible for receiving $S-1$ adjuvant chemotherapy $(n=503)$

$$
\begin{aligned}
& \text { Reasons for not administering } \mathrm{S}-1(\mathrm{n}=102) \\
& \text { 1) Patients attending physicians had determined ineligible }(\mathrm{n}=34) \\
& \text { 2) Refusal }(\mathrm{n}=21) \\
& \text { 3) Others }(\mathrm{n}=21) \\
& \text { 4) Unknown }(\mathrm{n}=26)
\end{aligned}
$$

Patients who received $S-1$ adjuvant chemotherapy $(n=401)$

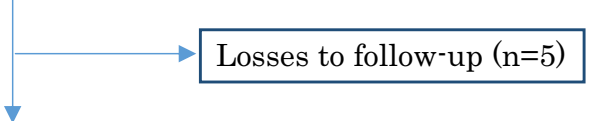

Follow-up (n=396 [Stage IIA/IIB//IIIA/IIIB/IIIC: 25/116/78/83/94])

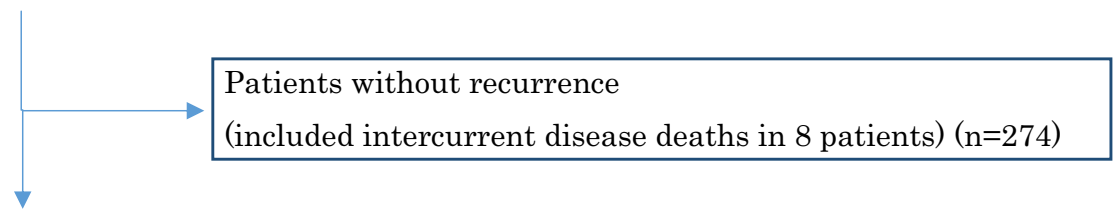

Patients with recurrence (n=122 [Stage IIA/IIB//IIIA/IIIB/IIIC: 3/18/16/29/56]) 
received preoperative chemotherapy. Of 503 patients, 401 were administered S-1 as adjuvant chemotherapy, which was intended to be continued for 1 year according to the results of the ACTS-GC [3, 4]. The major reasons for not administering S-1 to 102 other patients were as follows: physician's discretion, patient's refusal, administration of other regimens or treatment of other diseases. Although 5 of 401 patients were lost to follow-up, the vital status of each of the remaining 396 was acquired. Their median follow-up was 60 months [range 5-105 months, interquartile range (IQR) 51-65].

Regular visits to the hospital were made by 396 patients for 5 years after gastrectomy. Patients were evaluated using assays for serum tumor markers every 3 months for the first 2 years after gastrectomy and every 6 months for the remaining 3 years. Whole-body computed tomography was performed every 6 months. The remnant stomach was endoscopically examined during the first, third and fifth postoperative years.

The study included 122 of 396 (30.8\%) patients who suffered from cancer recurrence during follow-up. Recurrence was confirmed using at least image analysis, and histological confirmation was not required. Only elevated levels of tumor markers were not included in the analysis of recurrence.

All descriptions of gastric cancer were based on the Japanese classification of gastric carcinoma: 3rd English edition [10]. The cut-off date for acquiring data was April 30, 2018. The institutional review boards of the Cancer Institute Hospital approved this study.

\section{Adjuvant therapy}

S-1 monotherapy was planned and administered according to the standard protocol used in the ACTS-GC as follows: $80-120 \mathrm{mg} / \mathrm{day}$ of S-1 for 4 weeks, 2 weeks of rest, therapy continued for 1 year after surgery or eight courses. Dose or schedule modifications of S-1 because of adverse events or patients' condition were made mainly according to the standardized methods described in the ACTS-GC protocol. We defined completion of initial S-1 therapy as continuing S-1 on the day 1 year after surgery, regardless of the number of courses or total dose. Otherwise, cases were designated as discontinuation of S-1, regardless of the reasons.

\section{Analysis}

We analysed the clinicopathological characteristics of patients with recurrence. RFS was calculated to determine when disease recurred, and RFS rates among pathological substages were compared. RFS was defined as the time from curative surgery to cancer recurrence or death. Postrecurrence OS was defined as the time from confirmation of recurrence to death or to the last date when the patient was confirmed to be alive. The sites of first relapse have divided the groups as follows: local recurrence, lymph node metastasis, peritoneal dissemination and hematogenous metastasis. Ovarian metastases were included in peritoneal dissemination.

To determine the trend of each recurrence, the cumulative incidence was calculated, and the curves were generated according to each recurrent site.

\section{Statistical analysis}

Statistical calculations were carried out using EZR (Saitama Medical Center, Jichi Medical University, Saitama, Japan) [11], and survival was calculated using the Kaplan-Meier method. A log-rank test was used to compare survival between groups. $P$ values $<0.05$ were considered to indicate statistical significance. The number of patients with stage II was small. Therefore, patients with stage IIA or IIB disease were combined into stage II.

\section{Results}

\section{Patients' characteristics}

Among the 122 patients who experienced a recurrence, tumor stages were as follows: pStages IIA, IIB, IIIA, IIIB and IIIC in 3, 18, 16, 29 and 56 patients, respectively. The frequencies of recurrence during follow-up of 396 patients were as follows: $12.0 \%$, pStage IIA; $15.5 \%$, pStage IIB; $20.5 \%$, pStage IIIA; $34.9 \%$, pStage IIIB; and 59.6\%, pStage IIIC. Patients' characteristics are summarized in Table 1. Among the 122 patients who experienced recurrence, $90(73.7 \%)$ had pT4 disease and 71 (58.2\%) had pN3 disease. Therefore, 85 (69.7\%) patients with a recurrence had stage IIIB or IIIC disease.

Medication histories were not available for two patients because they were administered chemotherapy at another hospital. Of 120 patients with a recurrence, the median duration of adjuvant S-1 monotherapy was 10 months (range 0-15 months, IQR 4-10). Adjuvant S-1 monotherapy was administered to $85(70.8 \%)$ patients for more than 6 months, $79(65.8 \%)$ completed a 1-year course, 22 experienced a recurrence during chemotherapy and disease recurred in 14 patients soon after they completed 1 year of treatment. One-year therapy was incomplete for 41 (34.2\%) patients for the reasons as follows: $n=22$, recurrences as described above; $n=13$, gastrointestinal toxicity; $n=3$, hematological adverse events; $n=1$, eczema; $n=1$, ophthalmopathy and $n=1$ patient's refusal. 
Table 1 Characteristics of 122 patients with recurrence after radical gastrectomy followed by S-1 adjuvant chemotherapy

\begin{tabular}{lc}
\hline Variable & $\begin{array}{c}\text { Number of } \\
\text { patients }(\%)\end{array}$ \\
\hline Sex & \\
Male & $84(68.9)$ \\
Female & $38(31.1)$ \\
Median age, years (IQR) & $63(56-71)$ \\
Surgical procedure & \\
Total gastrectomy & $56(45.9)$ \\
Distal gastrectomy & $66(54.1)$ \\
Surgical approach & \\
Open & $116(95.1)$ \\
Laparoscopic & $6(4.9)$ \\
Histological type & \\
Differentiated & $42(34.4)$ \\
Undifferentiated & $80(65.6)$ \\
T factor & \\
T2 & $10(8.2)$ \\
T3 & $22(18.0)$ \\
T4a & $83(68.0)$ \\
T4b & $7(5.7)$ \\
N factor & \\
N0 & $10(8.2)$ \\
N1 & $19(15.6)$ \\
N2 & $22(18.0)$ \\
N3 & $71(58.2)$ \\
IIA & \\
IIB & $10(14.8)$ \\
IIIA & $29(23.8)$ \\
Median duration of S-1, months (IQR) & $56(45.9)$ \\
\hline & \\
III & $10(40)$ \\
\hline
\end{tabular}

$I Q R$ interquartile range
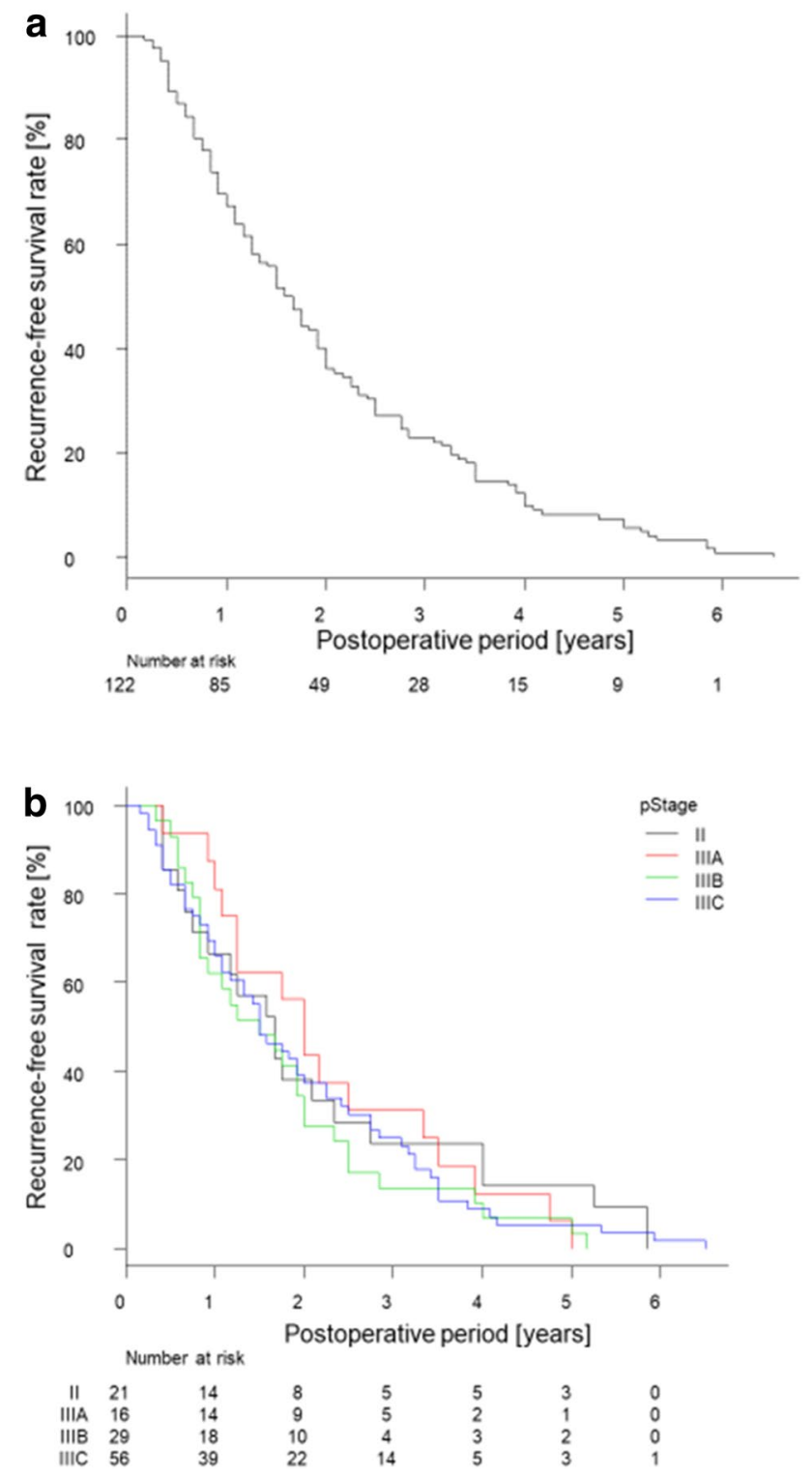

Fig. 2 Kaplan-Meier analyses of recurrence and survival. a Recurrence-free survival (RFS) of patients who underwent radical gastrectomy followed by S-1 adjuvant chemotherapy. b RFS according to the staging of patients with recurrence after radical gastrectomy followed by $S-1$ adjuvant chemotherapy

\section{Sites of recurrence}

The sites of initial recurrence were as follows: 63, peritoneal; 42, hematogenous; 25 , lymphatic and 6 , local. The most frequent site of hematogenous metastasis was the liver, $(n=22)$, and 13 patients $(10.7 \%)$ experienced recurrences at two sites (Fig. 3). Patients with lymphatic metastasis tended to present with contemporaneous peritoneal and hematogenous metastasis. Only one patient experienced three overlapping sites. 


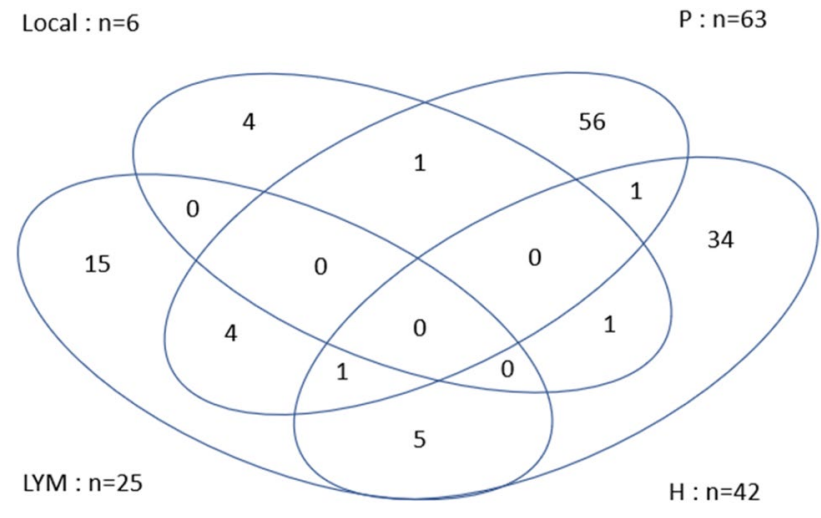

Local, local recurrence; $P$, peritoneal dissemination;

LYM, Iymph node metastasis; $\mathrm{H}$, hematogenous metastasis.

Fig. 3 Overall pattern of relapse of 122 patients with recurrence after radical gastrectomy followed by S-1 adjuvant chemotherapy. The sites of the first relapse have divided the groups as follows: Local local recurrence, $P$ peritoneal dissemination, $L Y M$ lymph node metastasis, $H$ hematogenous metastasis

\section{Trends of recurrence}

The numbers of local (100\%) and lymphatic (92.0\%) recurrences plateaued 3 years after gastrectomy (Fig. 4a). In contrast, the numbers of patients with peritoneal and hematogenous recurrence continued to increase within 5 years after surgery. When hematogenous recurrences were divided into liver metastasis and others, liver metastasis plateaued in 3 years, and the numbers of the others increased with time (Fig. 4b).

\section{Survival after confirmation of recurrence}

The median post-recurrence OS was 10 months (range 0-100 months, IQR 5-19.5) (Fig. 5a). Post-recurrence survival curves according to substage are shown in Fig. 5 b. The pathological substage did not significantly affect postrecurrence OS $[P=0.311]$.

\section{Discussion}

Here, we present three new findings about timing and trends of recurrence of gastric cancer patients with pStage II or III disease who underwent adjuvant S-1 monotherapy following curative gastrectomy. First, $80 \%$ of recurrences occurred within 3 years after surgery, although there were no significant differences between recurrences and pathological substages. Second, trends of recurrence were site-specific. Third, the median survival time (MST) after confirmation of a recurrence was 10 months, and there was no significant association between the duration of survival and

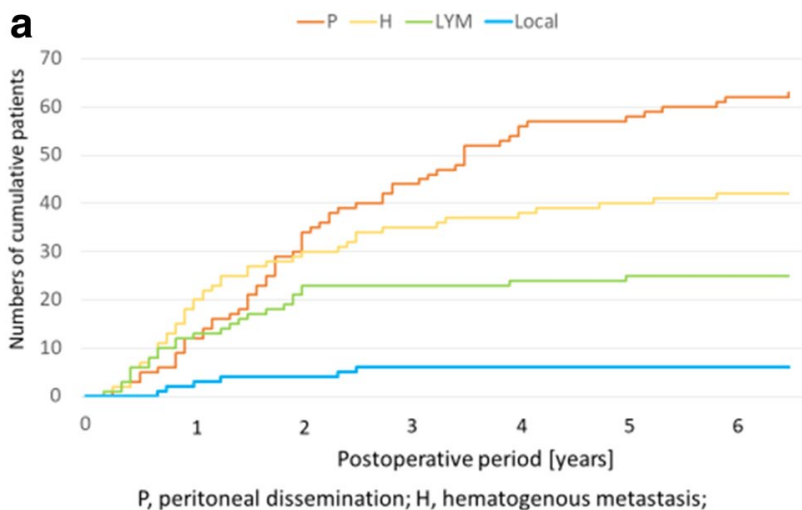

$\mathrm{P}$, peritoneal dissemination; $\mathrm{H}$, hematogenous metastasis; LYM, lymph node metastasis; Local, local recurrence.

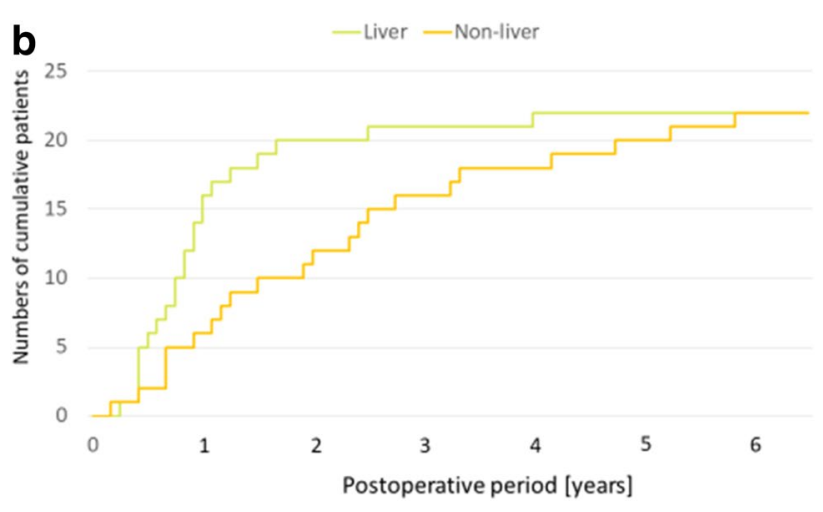

Fig. 4 Analyses of sites of recurrence. a Cumulative numbers of patients with peritoneal dissemination, hematogenous metastasis, lymph node metastasis and local recurrence. b Cumulative numbers of patients with hematogenous metastasis divided into liver metastasis and non-liver metastasis groups. $P$ peritoneal dissemination, $H$ hematogenous metastasis, LYM lymph node metastasis, Local local recurrence

pathological substages. These new findings may serve as a useful reference for managing pStage II or III gastric cancer patients undergoing curative gastrectomy followed by adjuvant S-1 monotherapy.

Adjuvant S-1 monotherapy prolonged the time until a recurrence was detected. Nashimoto et al. reported that among the patients with recurrence who were not treated using adjuvant chemotherapy for advanced gastric cancer in Japan, $79.2 \%$ and $90.7 \%$ experienced a recurrence within 2 and 3 years after radical surgery [7]. D'Angelica et al. also reported that $79 \%$ of patients with a recurrence were detected within 2 years after surgery [8], which included small numbers of patients with early gastric cancer that were reported to recur later than those with advanced gastric cancer [12, 13]. Times to recurrence reported here (2- and 3-year RFS rates were $36.1 \%$ and $23.0 \%$, respectively) were longer compared with those studies described above. S-1 may suppress the growth of micrometastasis in some patients for 1 year. Thus, we propose to amend the recommendation of the guidelines on the surveillance for pStage II or III gastric 

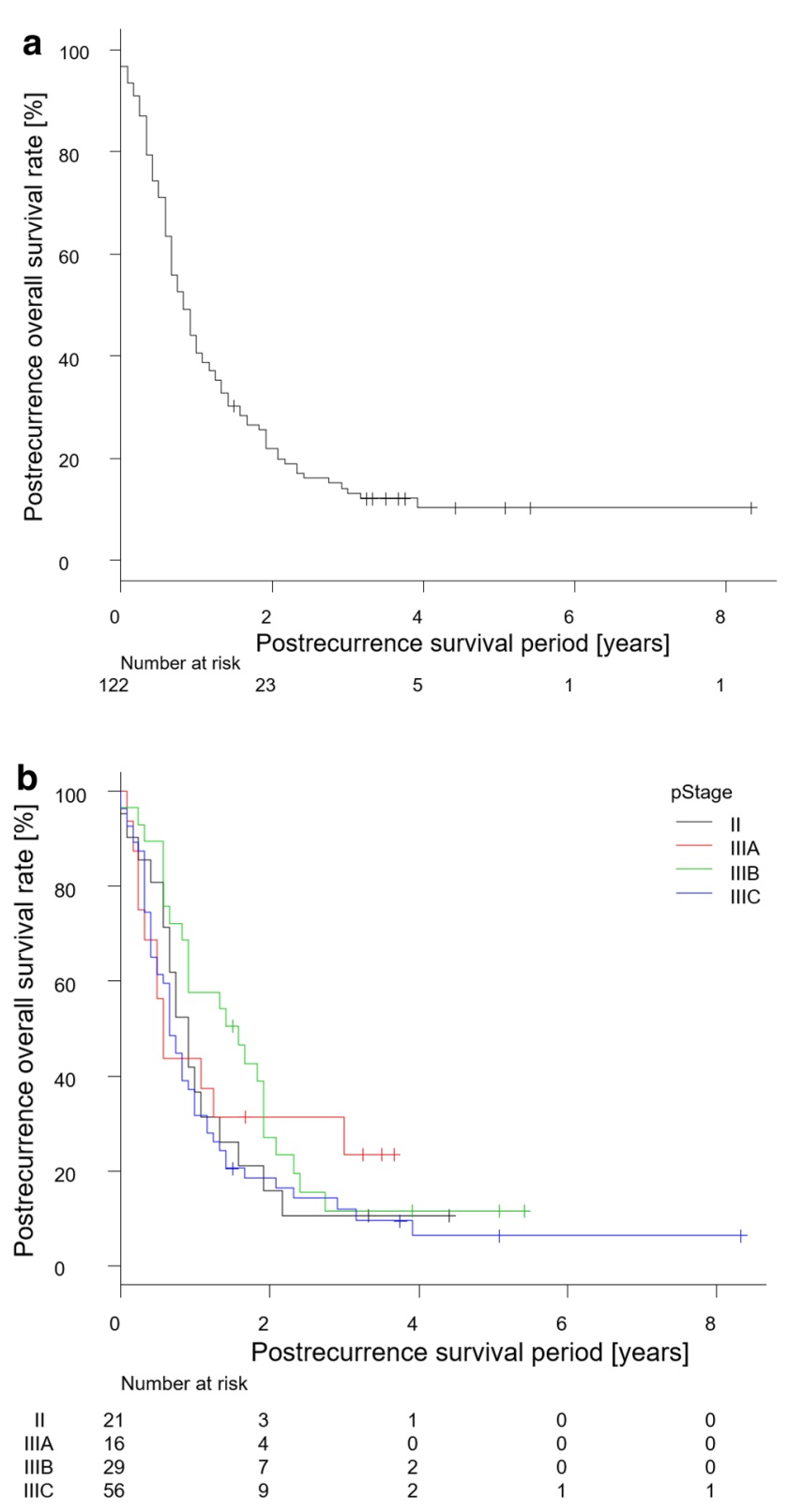

Fig. 5 Kaplan-Meier analyses of post-recurrence survival. a Survival of patients after recurrence was confirmed. b Survival according to the staging of patients after confirmation of recurrence

cancer patients undergoing curative gastrectomy. As such patients usually receive adjuvant chemotherapy, we should survey them every 3 months for at least 3 years after surgery while the guidelines recommend us to make a survey every 3 months for 2 years [5]. Furthermore, physicians should take care to consider that $20 \%$ of recurrent patients experience recurrence more than 3 years after surgery followed by adjuvant S-1 monotherapy.

We initially supposed that patients with earlier substages would experience recurrence later than those with a more advanced substage. For example, evidence indicates that recurrence is experienced by patients with early gastric cancer later compared with those with advanced disease [12, 13]. However, the interval between surgery and recurrence was not significantly affected by the pathological substage. Only the frequency of recurrence depended on pathological substages. These results indicate that it may take the same time for S-1-resistant cancer cells to form a visible tumor, which is independent of substage. Surgical quality in a highvolume center that achieves successful radical gastrectomy for all stages may contribute to this result [6]. However, there are no reports to our knowledge about the relationship between pathological substages and time-to-recurrence. Therefore, it is not possible to determine whether the results reflect a specific feature of adjuvant S-1 monotherapy.

The profile of sites of recurrence reported here is quite similar to that of the S-1 cohort of the ACTS-GC [3, 4], which supports the conclusion that the results of our present study are valid. It is important to note that we are the first to report trends in recurrence experienced by patients receiving adjuvant S-1 monotherapy. Specifically, we discovered two patterns of recurrence. First, the incidences of local recurrence and lymph node metastasis plateaued 3 years after gastrectomy. Second, peritoneal and hematogenous metastases increased within 5 years.

Local recurrence and lymph node metastasis were easy to detect using imaging. In contrast, peritoneal metastasis was indicated by patients' symptoms. These different diagnostic techniques may affect the trend. For example, approximately $50 \%$ of hematogenous recurrences localized to the liver and were easy to detect. However, the other half included metastasis to bone, lung or adrenal gland, which may be overlooked by routine imaging examinations. These results suggest that physicians should be aware of recurrence patterns 3 years after gastrectomy and that they should pay particularly close attention to symptoms associated with peritoneal metastasis or hematogenous metastasis other than liver metastasis. Future improvements in diagnostic techniques, such as staging laparoscopy, and postoperative management, may favorably alter the trends reported here.

The MST after confirmation of recurrence was 10 months. Ito et al. reported that the median time from recurrence to death was 11.4 months in the adjuvant S-1 group using the data from patients enrolled in the ACTS-GC, and adjuvant chemotherapy with S-1 did not influence the time from recurrence to death [14]. Similarly, the present data were inferior compared with those of certain clinical trials that reported the survival periods were 10.1-13.8 months after recurrence [15-18]. As a result of clinical practice including various kinds of patients, 10 months survival might be clinically expected.

Our study had certain limitations. The number of subjects was 122 . These patients were included with those who were closely followed. Although the total size of this group was 
relatively large for a study such as this, the number in each subgroup was very small when every substage or site of recurrence was analysed. Thus, the results of this study are not definitive, although it is the largest such study conducted in Japan at a single institution. Further, we did not investigate the relationship between dose intensity of S-1 adjuvant chemotherapy and the trends of recurrence, which must be analysed in the future.

In conclusions, $80 \%$ of recurrences of pStage II or III gastric cancer after adjuvant S-1 monotherapy occur within 3 years after curative gastrectomy, independent of pathological substages. Local recurrence, lymph node and liver metastasis are infrequent after 3 years, although peritoneal and certain types of hematogenous metastasis increase each year, even after 3 years. Survival after confirmation of recurrence is still poor as the MST is 10 months, which is not associated with substages. More effective agent and strategy should be developed immediately.

Acknowledgements Dr. Daisuke Takahari, Dr. Takashi Ichimura, Dr. Mariko Ogura and Dr. Takeru Wakatsuki of the Department of Gastroenterology, Cancer Institute Hospital, Japanese Foundation for Cancer Research treated the patients, and we thank them for collecting data.

\section{Compliance with ethical standards}

Conflict of interest The authors declare no conflicts of interest.

Ethical standards All procedures were conducted in accordance with the ethical standards of institutional and national committees responsible for human experimentation and with the 1964 and later versions of the Declaration of Helsinki.

\section{References}

1. Ferlay J, Soerjomataram I, Dikshit R, Eser S, Mathers C, Rebelo $\mathrm{M}$, et al. Cancer incidence and mortality worldwide: sources, methods and major patterns in GLOBOCAN 2012. Int J Cancer. 2015;136(5):E359-86.

2. Nashimoto A, Akazawa K, Isobe Y, Miyashiro I, Katai H, Kodera Y, et al. Gastric cancer treated in 2002 in Japan: 2009 annual report of the JGCA nationwide registry. Gastric Cancer. 2013;16(1):1-27.

3. Sakuramoto S, Sasako M, Yamaguchi T, Kinoshita T, Fujii M, Nashimoto A, et al. Adjuvant chemotherapy for gastric cancer with S-1, an oral fluoropyrimidine. N Engl J Med. 2007;357(18):1810-20.

4. Sasako M, Sakuramoto S, Katai H, Kinoshita T, Furukawa $\mathrm{H}$, Yamaguchi T, et al. Five-year outcomes of a randomized phase III trial comparing adjuvant chemotherapy with S-1 versus surgery alone in stage II or III gastric cancer. J Clin Oncol. 2011;29(33):4387-93.
5. Japanese gastric cancer treatment guidelines 2014 (Ver. 4). Gastric Cancer. 2017;20(1):1-19.

6. Kano Y, Ohashi M, Hiki N, Takahari D, Chin K, Yamaguchi K, et al. Favorable long-term outcomes of one-year adjuvant S-1 monotherapy for pathological stage II or III gastric cancer treated at a high-volume center. Gastric Cancer. 2018.

7. Nashimoto A, Yabusaki H, Nakagawa S. Proper follow-up schedule after curative gastric surgery. Gan to kagaku ryoho Cancer Chemotherapy. 2009;36(9):1402-7.

8. D' Angelica M, Gonen M, Brennan MF, Turnbull AD, Bains M, Karpeh MS. Patterns of initial recurrence in completely resected gastric adenocarcinoma. Ann Surg. 2004;240(5):808-16.

9. Baiocchi GL, Kodera Y, Marrelli D, Pacelli F, Morgagni P, Roviello F, et al. Follow-up after gastrectomy for cancer: results of an international web round table. World J Gastroenterol. 2014;20(34):11966-71.

10. Japanese classification of gastric carcinoma. 3rd English edition. Gastric Cancer. 2011;14(2):101-12.

11. Kanda Y. Investigation of the freely available easy-to-use software 'EZR' for medical statistics. Bone Marrow Transplant. 2013;48(3):452-8.

12. Saka M, Katai H, Fukagawa T, Nijjar R, Sano T. Recurrence in early gastric cancer with lymph node metastasis. Gastric Cancer. 2008;11(4):214-8.

13. Yamamoto M, Yamanaka T, Baba H, Kakeji Y, Maehara Y. The postoperative recurrence and the occurrence of second primary carcinomas in patients with early gastric carcinoma. J Surg Oncol. 2008;97(3):231-5.

14. Ito S, Ohashi Y, Sasako M. Survival after recurrence in patients with gastric cancer who receive S-1 adjuvant chemotherapy: exploratory analysis of the ACTS-GC trial. BMC Cancer. 2018;18(1):449.

15. Koizumi W, Narahara H, Hara T, Takagane A, Akiya T, Takagi M, et al. S-1 plus cisplatin versus S-1 alone for first-line treatment of advanced gastric cancer (SPIRITS trial): a phase III trial. Lancet Oncol. 2008;9(3):215-21.

16. Boku N, Yamamoto S, Fukuda H, Shirao K, Doi T, Sawaki A, et al. Fluorouracil versus combination of irinotecan plus cisplatin versus S-1 in metastatic gastric cancer: a randomised phase 3 study. Lancet Oncol. 2009;10(11):1063-9.

17. Bang YJ, Van Cutsem E, Feyereislova A, Chung HC, Shen L, Sawaki A, et al. Trastuzumab in combination with chemotherapy versus chemotherapy alone for treatment of HER2-positive advanced gastric or gastro-oesophageal junction cancer (ToGA): a phase 3, open-label, randomised controlled trial. Lancet. 2010;376(9742):687-97.

18. Ohtsu A, Shah MA, Van Cutsem E, Rha SY, Sawaki A, Park $\mathrm{SR}$, et al. Bevacizumab in combination with chemotherapy as first-line therapy in advanced gastric cancer: a randomized, double-blind, placebo-controlled phase III study. J Clin Oncol. 2011;29(30):3968-76.

Publisher's Note Springer Nature remains neutral with regard to jurisdictional claims in published maps and institutional affiliations. 